\title{
EXPANSION FORMULAE FOR GENERAL TRIPLE HYPERGEOMETRIC SERIES
}

\author{
CH. WALI MOHD AND M. I. QURESHI ${ }^{1}$
}

(Received 11 July 1984; revised 27 March 1985)

\begin{abstract}
The main object of present paper is to obtain a finite summation of Srivastava's general triple hypergeometric series in terms of Kampé de Fériet's double hypergeometric series. A number of finite sums of Kampé de Fériet's double hypergeometric polynomials in terms of different kinds of single hypergeometric polynomials of higher order, are obtained. Some known results of Manocha and Sharma [9], [10], Munot [11], Pathan [12], Qureshi [15], Qureshi and Pathan [16] and Srivastava [26] are deduced as special cases. A result of Pathan [13, page 316 (1.2)] is also corrected here.
\end{abstract}

\section{Introduction}

A unification of Lauricella's fourteen triple hypergeometric functions $F_{1}, \ldots, F_{14}$ [7, pages 113-114], the extended $F_{k}$ function of Sharma [20, page 613 (2)] and three additional functions $H_{A}, H_{B}$ and $H_{C}$ of Srivastava [22, pages 99-100, see also 23 and 25] was introduced by Srivastava [24, page 428] in the form of a general triple hypergeometric series $F^{(3)}$, defined as

$$
\begin{gathered}
F^{(3)}\left[\begin{array}{l}
(a)::(b) ;\left(b^{\prime}\right) ;\left(b^{\prime \prime}\right):(c) ;\left(c^{\prime}\right) ;\left(c^{\prime \prime}\right) ; \quad x, y, z \\
(d)::(e) ;\left(e^{\prime}\right) ;\left(e^{\prime \prime}\right):(f) ;\left(f^{\prime}\right) ;\left(f^{\prime \prime}\right) ;
\end{array}\right] \\
=\sum_{m, n, p=0}^{\infty} \frac{[a]_{m+n+p}[b]_{m+n}\left[b^{\prime}\right]_{n+p}\left[b^{\prime \prime}\right]_{p+m}}{[d]_{m+n+p}[e]_{m+n}\left[e^{\prime}\right]_{n+p}\left[e^{\prime \prime}\right]_{p+m}} \\
\cdot \frac{[c]_{m}\left[c^{\prime}\right]_{n}\left[c^{\prime \prime}\right]_{p}}{[f]_{m}\left[f^{\prime}\right]_{n}\left[f^{\prime \prime}\right]_{p}} \frac{x^{m}}{m !} \frac{y^{n}}{n !} \frac{z^{p}}{p !}
\end{gathered}
$$

\footnotetext{
${ }^{1}$ Department of Mathematics, Aligarh Muslim University, Aligarh-202001, India.

(1) Copyright Australian Mathematical Society 1986, Serial-fee code 0334-2700/86
} 
where $(a)$ is the abbreviation for the array of $A$ parameters $a_{1}, a_{2}, \ldots, a_{A}$. It is to be noted here that there are $A$ of the (a) parameters, $B$ of the $(b)$ parameters and so on. Thus $[a]_{m}$ is interpreted as

$$
[a]_{m}=\prod_{i=1}^{A}\left(a_{i}\right)_{m}=\prod_{i=1}^{A} \frac{\Gamma\left(a_{i}+m\right)}{\Gamma\left(a_{i}\right)}
$$

with similar interpretations for $[b],[c]$, et cetera. It will be assumed throughout the paper that the absence of parameters shown by horizontal dashes mean that there exists no parameter, and in that case, from (1.2), the conventional value of an empty product will be unity, i.e.,

$$
\prod_{i=1}^{0}\left(a_{i}\right)_{m}=1
$$

Also, numerator parameters like $(a),(b),\left(b^{\prime}\right),\left(b^{\prime \prime}\right)$ et cetera may be zero or negative integers, but the denominator parameters like $(d),(e)$ et cetera are not allowed to be zero or negative integers.

The region of convergence of the above triple power series (1.1) is given in the recent literature [3, page 156; see also 4, page 40].

The main object of the present paper is to obtain the following finite sum of Srivastava's general triple hypergeometric series in terms of Kampé de Fériet's double hypergeometric series [1, page 150]

$$
\begin{aligned}
\sum_{n=0}^{m} \frac{(-m)_{n}}{n !} F^{(3)}\left[\begin{array}{l}
\left(a^{\prime}\right)::(a) ;\left(g^{\prime}\right) ;-:-n,(d) ;-m+n,(g) ;(c) ; \\
\left(b^{\prime}\right)::(b) ;\left(h^{\prime}\right) ;-:(e) ;(h) ;(f) ;
\end{array}\right] \\
\quad=\frac{x^{m}[a]_{m}\left[a^{\prime}\right]_{m}[d]_{m}}{[b]_{m}\left[b^{\prime}\right]_{m}[e]_{m}} \\
\cdot F\left[\begin{array}{l}
\left(g^{\prime}\right):\left(a^{\prime}+m\right),(c) ;-m,(1-e-m),(g) ; \\
\left(h^{\prime}\right):\left(b^{\prime}+m\right),(f) ;(1-d-m),(h) ;
\end{array}\right]
\end{aligned}
$$

where each of the parameters $(d)=\left\{d_{1}, d_{2}, \ldots, d_{D}\right\}$ need not be an integer. Also the variable $x$ should not be zero. The notation used here for Kampé de Fériet's double series is due to Burchnall and Chaundy [2, page 112]. 
Some finite sums for hypergeometric polynomials of Krall and Frink, Rainville, Gegenbauer, generalized Rice, Shively's Pseudo-Laguerre, Bateman, Generalized Laguerre and Jacobi are deduced as special cases of (1.3). A number of known results are also obtained from our main result in Section 3.

\section{Proof of the main result (1.3)}

Consider the series

$$
\begin{aligned}
\mathrm{S}= & \sum_{n=0}^{m} \frac{(-m)}{n !} F^{(3)}\left[\begin{array}{l}
\left.\left(a^{\prime}\right)::(a) ;\left(g^{\prime}\right) ;-:-n,(d) ;-m+n,(g) ;(c) ; x, y, z\right] \\
\left(b^{\prime}\right)::(b) ;\left(h^{\prime}\right) ;-:(e) ;(h) ;(f) ;
\end{array}\right] \\
= & \sum_{n=0}^{m} \frac{(-m)_{n}}{n !} \sum_{p=0}^{\infty} \frac{\left[a^{\prime}\right]_{p}\left[g^{\prime}\right]_{p}[c]_{p}}{\left[b^{\prime}\right]_{p}\left[h^{\prime}\right]_{p}[f]_{p}} \frac{z^{p}}{p !} \\
& \sum_{r=0}^{n} \sum_{s=0}^{m-n} \frac{[a]_{r+s}\left[a^{\prime}+p\right]_{r+s}[d]_{r}[g]_{s}\left[g^{\prime}+p\right]_{s}}{[b]_{r+s}\left[b^{\prime}+p\right]_{r+s}[e]_{r}[h]_{s}\left[h^{\prime}+p\right]_{s}}(-n)_{r}(n-m)_{s} \frac{x^{r}}{r !} \frac{y^{s}}{s !}
\end{aligned}
$$

Now using the finite triple series identity of Srivastava [26, page 95]

$$
\sum_{n=0}^{m} \sum_{r=0}^{n} \sum_{s=0}^{m-n} A(m, n, r, s)=\sum_{s=0}^{m} \sum_{r=0}^{m-s} \sum_{n=0}^{m-r-s} A(m, n+r, r, s)
$$

and many Pochhammer's identities like

$$
\left.\begin{array}{l}
{[a]_{m}=(-1)^{m A}[1-a-m]_{m}} \\
{[b]_{m}=[b]_{s}[b+s]_{m-s}} \\
{[d]_{m-s}=[d]_{m} \frac{(-1)^{s D}}{[1-d-m]_{s}}} \\
(-n-s)_{s}=(-1)^{s} \frac{(n+s) !}{n !}
\end{array}\right\},
$$

we have

$$
\begin{aligned}
& \mathrm{S}= m ! \sum_{p=0}^{\infty} \frac{\left[a^{\prime}\right]_{p}\left[g^{\prime}\right]_{p}[c]_{p}}{\left[b^{\prime}\right]_{p}\left[h^{\prime}\right]_{p}[f]_{p}} \frac{z_{p}^{p}}{p !} \sum_{s=0}^{m} \frac{\left[a^{\prime}+p\right]_{s}[a]_{s}\left[g^{\prime}+p\right]_{s}[g]_{s}}{[b]_{s}\left[b^{\prime}+p\right]_{s}[h]_{s}\left[h^{\prime}+p\right]_{s}} \frac{(-y)^{s}}{s !} \\
& \cdot \sum_{r=0}^{m-s} \frac{\left[a^{\prime}+p+s\right]_{r}[a+s]_{r}[d]_{r} x^{r}}{[b+s]_{r}\left[b^{\prime}+p+s\right]_{r}[e]_{r} r !(m-r-s) !} \sum_{n=0}^{m-r-s}(-1)^{n}(m-r-s \\
& {[m}
\end{aligned} .
$$


Now using the combinatorial identity

$$
\sum_{n=0}^{k}(-1)^{n}\left(\begin{array}{l}
k \\
n
\end{array}\right)= \begin{cases}1, & \text { if } k=0, \\
0, & \text { if } k=1,2,3, \ldots,\end{cases}
$$

in (2.4), we find that the value of the $r$-summation reduces to that of its last term, all other terms vanishing.

So for $r=(m-s)$, we have

$$
\begin{aligned}
\mathrm{S}= & \frac{[a]_{m}\left[a^{\prime}\right]_{m}[d]_{m} x^{m}}{[b]_{m}\left[b^{\prime}\right]_{m}[e]_{m}} \\
& \sum_{p=0}^{\infty} \sum_{s=0}^{m} \frac{\left[g^{\prime}\right]_{p+s}\left[a^{\prime}+m\right]_{p}[c]_{p}(-m)_{s}[1-e-m]_{s}[g]_{s}}{\left[h^{\prime}\right]_{p+s}\left[b^{\prime}+m\right]_{p}[f]_{p}[1-d-m]_{s}[h]_{s}} \\
& \frac{(-1)^{s(D-E)}}{s !}\left(\frac{y}{x}\right)^{s} \frac{z^{p}}{p !}
\end{aligned}
$$

which reduces to our main result (1.3)

\section{Cases of reducibility}

When $y=0$ and $A=B=G^{\prime}=H^{\prime}=0$ in (1.3), we have

$$
\begin{aligned}
\sum_{n=0}^{m} \frac{(-m)_{n}}{n !} F\left[\begin{array}{ll}
\left(a^{\prime}\right):-n,(d) ;(c) ; & \\
\left(b^{\prime}\right):(e) ;(f) ; & x, z
\end{array}\right] \\
\quad=\frac{\left[a^{\prime}\right]_{m}[d]_{m} x^{m}}{\left[b^{\prime}\right]_{m}[e]_{m}}{ }_{A^{\prime}+C} F_{B^{\prime}+F}\left[\begin{array}{ll}
\left(a^{\prime}+m\right),(c) ; & z \\
\left(b^{\prime}+m\right),(f) ;
\end{array}\right] .
\end{aligned}
$$

When $z=0$ and $A^{\prime}=B^{\prime}=G^{\prime}=H^{\prime}=0$ in (1.3), we have

$$
\begin{aligned}
& \sum_{n=0}^{m} \frac{(-m)_{n}}{n !} F\left[\begin{array}{l}
(a):-n,(d) ;-m+n,(g) ; x, y \\
(b):(e) ;(h) ;
\end{array}\right] \\
& =\frac{[a]_{m}[d]_{m} x^{m}}{[b]_{m}[e]_{m}}{ }_{1+E+G} F_{D+H}\left[\begin{array}{l}
-m,(1-e-m),(g) ; \\
(1-d-m),(h) ;
\end{array} \quad(-1)^{D-E} \frac{y}{x}\right] .
\end{aligned}
$$


Setting $A=E=H=1$ and $B=D=G=0$ in (3.2), and making suitable adjustment of parameters, using the definition of Jacobi's polynomials [18, page 255 (8)], we have a known result of Munot [11, page 691 (2.1); see also 26, page 95 (7.13)]

$$
\begin{aligned}
& \sum_{n=0}^{m} \frac{(-b-m)_{n}(1+b)_{m-n}}{n !(m-n) !} F_{2}[d ;-n, n-m ; 1+a, 1+b ; x, y] \\
& \quad=\frac{(d)_{m}(-1)^{m}}{(1+a)_{m}}(x+y)^{m} P_{m}^{(a, b)}\left(\frac{y-x}{y+x}\right),
\end{aligned}
$$

where $F_{2}$ is Appell's polynomial of second kind [5, page 224 (7)].

In fact, the result (3.3) was obtained by Munot [11] using operational calculus techniques, while Srivastava [26, page 95] derived the same result using method of series manipulation.

On taking $A=B=0$ in (3.2), we have a known result of Manocha and Sharma [10, page $233(16)]$

$$
\begin{aligned}
& \sum_{n=0}^{m} \frac{(-m)_{n}}{n !}{ }_{D+1} F_{E}\left[\begin{array}{lr}
-n,(d) ; & \\
(e) ; & x
\end{array}\right]_{G+1} F_{H}\left[\begin{array}{ll}
-m+n,(g) ; & \\
(h) ;
\end{array}\right] \\
& \quad=\frac{[d]_{m} x^{m}}{[e]_{m}}{ }_{1+E+G} F_{D+H}\left[\begin{array}{l}
-m,(1-e-m),(g) ; \\
(1-d-m),(h) ;
\end{array}\right]
\end{aligned}
$$

which was obtained by the method of fractional derivative.

Taking $D=E=G=H=1$ in (3.4), we get a known result of Qureshi and Pathan [16, page 180 equation (3.5)].

Setting $A=A^{\prime}=B^{\prime}=C=E=H=1$ and $B=D=F=G=G^{\prime}=H^{\prime}=0$ in (1.3), adjusting parameters and variables suitably and using the definition of Jacobi's polynomials [18, page 255 (8)], we get a known result of Pathan [12, page $59(2.3)]$

$$
\begin{aligned}
& \sum_{n=0}^{m} \frac{(-m)_{n}}{n !} F^{(3)}\left[\begin{array}{l}
d:: a ;-;-:-n ;-m+n ; c ; \\
b::-;-;-: 1+e ; 1+h ;-;
\end{array} \quad x, y, z\right] \\
& =\frac{m !(-)^{m}(a)_{m}(d)_{m}}{(1+h)_{m}(b)_{m}(1+e)_{m}}(y+x)^{m}{ }_{2} F_{1}\left[\begin{array}{ll}
d+m, c ; \\
b+m ;
\end{array}\right] P_{m}^{(e, h)}\left(\frac{y-x}{y+x}\right),
\end{aligned}
$$

which was obtained by the method of operational calculus. 
Again, setting $A^{\prime}=B^{\prime}=C=A=E=H=1, B=G^{\prime}=H^{\prime}=D=F=0$, changing $n, m$ into $m, n$ in (1.3) and making suitable adjustment of parameters and variables, we obtain

$$
\begin{aligned}
& \sum_{m=0}^{n} \frac{(-n)_{m}}{m !} F^{(3)}\left[\begin{array}{ll}
a:: b ;-;-:-m ; m-n ; c-b ; & \frac{x}{1+z}, \frac{-x}{1+z}, \frac{z}{1+z} \\
c::-;-;-: 1+e ; 1+h ;-; &
\end{array}\right] \\
& =\frac{(1+e+h)_{2 n}(a)_{n}(b)_{n}}{(1+e)_{n}(1+h)_{n}(1+e+h)_{n}(c)_{n}}\left(\frac{x}{1+z}\right)^{n}{ }_{2} F_{1}\left[\begin{array}{ll}
a+n, c-b ; & \frac{z}{1+z} \\
c+n ;
\end{array}\right],
\end{aligned}
$$

which is the correct form of the following result of Pathan [13, page $316(1.2)]$

$$
\begin{aligned}
& \sum_{m=0}^{n} \frac{(-h-n)_{m}(1+h)_{n-m}}{m !(n-m) !} \\
& F^{(3)}\left[\begin{array}{ll}
a:: b ;-;-:-m ; m-n ; c-b ; & \frac{x}{1+z}, \frac{-x}{1+z}, \frac{z}{1+z} \\
c::-;-;-: 1+e ; 1+h ;-; &
\end{array}\right] \\
& =\frac{(1+e+h)_{2 n}(a)_{n}(b)_{n}}{(1+e)_{n}(1+h)_{n}(1+e+h)_{n}(c)_{n}}\left(\frac{x}{1+z}\right)^{n} \\
& { }_{2} F_{1}\left[\begin{array}{ll}
a+n, c-b ; & \frac{z}{1+z} \\
c+n ; &
\end{array}\right] \text {. }
\end{aligned}
$$

Also, in (3.6) taking $z=0$ and $z=x$ in conjunction with the use of a transformation of Pathan [14, page 372 (1.3)] we may obtain two other correct forms of the results of Pathan [13, page $317(3.2,3.4)]$.

When $A=E=H=0$ and $B=D=G=1$ in (3.2), we have a known result of Qureshi [15, page 48 (3.3.13)].

$$
\begin{gathered}
\sum_{n=0}^{m} \frac{(-m)_{n}}{n !} F_{3}[-n,-m+n ; d, g ; b ; x, y] \\
=\frac{(d)_{m} x^{m}}{(b)_{m}} F_{1}\left[\begin{array}{ll}
-m, g ; & \frac{-y}{x} \\
1-d-m ; &
\end{array}\right],
\end{gathered}
$$

where $F_{3}$ is Appell's polynomial of third kind [5, page $224(8)$ ]. 
When $A=B=D=G=0, E=H=1$ in (3.2), using the definition of generalized Laguerre's polynomials [18, page 200 (1)] and Jacobi's polynomials [18, page $255(8)$ ], we get a known result of Manocha and Sharma [9, page 475 (33)]

$$
\begin{aligned}
\sum_{n=0}^{m} & \frac{(-h-m)_{n}}{(1+e)_{n}} L_{n}^{(e)}(x) L_{m-n}^{(h)}(y) \\
& =\frac{(-)^{m}}{(1+e)_{m}}(x+y)^{m} P_{m}^{(e, h)}\left(\frac{y-x}{y+x}\right) .
\end{aligned}
$$

The result (3.9) was also obtained by Qureshi and Pathan [16, page 181 equation (3.8)] using the process of confluence [8, page $48(\$ 3.5)]$.

Setting $A=E=H=D=0, B=G=1$ in (3.2), making suitable adjustment of parameters and variables, using definitions of Humbert function $\Theta_{1}[5$, page $226(25)]$ and thereafter using Krall and Frink's simple Bessel polynomials $y_{m}(z)$ and generalized Bessel polynomials $y_{m}(a, b, z)[6$; see also 18, pages 293-294 (1.2)] and Rainville's polynomials $\phi_{m}(c, z)$ [18, page 294 (3)], we get three more relations in the form

$$
\begin{aligned}
& \sum_{n=0}^{m} \frac{(-m)_{n}}{n !} \Theta_{1}\left[-m+n,-n ; 1+m ; b ; \frac{-z}{2}, x\right] \\
& \quad=\frac{x^{m}}{(b)_{m}} y_{m}\left(\frac{z}{x}\right), \\
& \sum_{n=0}^{m} \frac{(-m)_{n}}{n !} \Theta_{1}[-m+n,-n ; a+m-1 ; b ; z,-x c] \\
& \quad=\frac{(-x c)^{m}}{(b)_{m}} y_{m}\left(a, c, \frac{z}{x}\right),
\end{aligned}
$$

and

$$
\begin{aligned}
& \sum_{n=0}^{m} \frac{(-m)_{n}}{n !} \Theta_{1}[-m+n,-n ; c+m ; b ; y, x] \\
& \quad=\frac{m ! x^{m}}{(b)_{m}(c)_{m}} \phi_{m}\left(c, \frac{y}{x}\right) .
\end{aligned}
$$

In (3.2), putting $G=H=2, E=D=0$ and using the definition of generalized Rice polynomials $H_{m}^{(a . b)}[g, h, v]$ [27, page $73(1.1)$; see also 19], we have

$$
\begin{aligned}
& \sum_{n=0}^{m} \frac{(-m)_{n}}{n !} F\left[\begin{array}{l}
(a):-n ;-m+n, 1+a+b+m, g ; \\
(b) ;-; 1+a, h ;
\end{array}\right] \\
& \quad=\frac{m ! x^{m}[a]_{m}}{(1+a)_{m}[b]_{m}} H_{m}^{(a, b)}\left[g, h, \frac{y}{x}\right] .
\end{aligned}
$$


Setting $D=E=0, H=G=1$ in (3.2), making suitable adjustment of parameters and variables and then using the definition of Gegenbauer's polynomials $C_{m}^{g}(x)[18$, page $279(15)]$, we have

$$
\begin{aligned}
& \sum_{n=0}^{m} \frac{(-m)_{n}}{n !} F\left[\begin{array}{l}
(a):-n ;-m+n, 2 g+m ; \\
(b) ;-; g+\frac{1}{2} ;
\end{array}\right] \\
& \quad=\frac{m ! x^{m}[a]_{m}}{(2 g)_{m}[b]_{m}} C_{m}^{g}\left(\frac{x-2 y}{x}\right) .
\end{aligned}
$$

Similarly setting $G=E=D=0, H=1$ in (3.2) and using the definition of Shively's Pseudo-Laguerre polynomials $R_{m}(g, v)$ [21, page 54 (48)], we have

$$
\sum_{n=0}^{m} \frac{(-m)_{n}}{n !} F\left[\begin{array}{l}
(a):-n ;-m+n ; \\
(b):-; m+h ;
\end{array} \quad x, y\right]=\frac{m ! x^{m}(h)_{m}[a]_{m}}{(h)_{2 m}[b]_{m}} R_{m}\left(h, \frac{y}{x}\right) .
$$

When $D=G=E=0, H=2$ in (3.2), adjusting parameters and variables suitably and then using the definition of Bateman's polynomials $J_{m}^{(g, h)}(v)[17$, page 721], we obtain

$$
\begin{array}{r}
\sum_{n=0}^{m} \frac{(-m)_{n}}{n !} F\left[\begin{array}{l}
(a):-n ;-m+n ; \\
(b):-; g+1, h+\frac{g}{2}+1 ;
\end{array} x^{2}, y^{2}\right. \\
=\frac{x^{2 m+g}[a]_{m} m ! \Gamma(g+1) \Gamma\left(h+\frac{g}{2}+1\right)}{y^{g}[b]_{m} \Gamma\left(h+\frac{g}{2}+m+1\right)} J_{m}^{(g, h)}\left(\frac{y}{x}\right) .
\end{array}
$$

When $D=E=G=H=1$ in (3.2), adjusting parameters suitably and using the definition of Rainville's polynomials $\psi_{m}(h, g, x)$ [18, page 302 (ex. 3)], we have

$$
\begin{aligned}
& \sum_{n=0}^{m} \frac{(-m)_{n}}{n !} F\left[\begin{array}{l}
(a):-n, \frac{1}{2}+\frac{1}{2} g ;-m+n, \frac{1}{2}-\frac{1}{2} g ; \\
(b): h ; h ;
\end{array}\right] \\
& \quad=\frac{(-1)^{m}[a]_{m}}{[b]_{m}} x^{m} \psi_{m}\left(h, g, \frac{y}{x}\right) .
\end{aligned}
$$




\section{Acknowledgement}

We are highly obliged to Dr. M. A. Pathan for his sincere guidance and constant encouragement in the preparation of this paper.

The authors are also thankful to the referee for his valuable suggestions in the improvement of this paper.

\section{References}

[1] P. Appell et J. Kampé de Fériet, Fonctions hypergéométriques et hypersphériques: Polynômes d'Hermite (Gauthier-Villars et cie., Paris, 1926).

[2] J. L. Burchnall and T. W. Chaundy, "Expansions of Appell's double hypergeometric functions. II," Quart. J. Math. Oxford Ser. 12 (1974), 112-128.

[3] S. P. Chhabra and K. C. Rusia, "A transformation formula for a general hypergeometric function of three variables," Jñannabha 9/10 (1980), 155-159.

[4] V. L. Deshpande, "Certain formulas associated with hypergeometric function of three variables," Pure Appl. Math. Sci. 14 (1981), 39-45.

[5] A. Erdélyi, et al., Higher transcendental functions. I, (McGraw-Hill, New York, 1953).

[6] H. L. Krall and O. Frink, "A new class of orthogonal polynomials: the Bessel polynomials," Trans. Amer. Math. Soc. 65 (1949), 100-115.

[7] G. Lauricella, "Sulle funzioni ipergeometriche a più variabili," Rend. Circ. Mat. Palermo 7 (1893), 111-158.

[8] Y. L. Luke, The special functions and thetr approxımations. I, (Academic Press, New York and London, 1969).

[9] H. L. Manocha and B. L. Sharma, "Summation of infinite series," J. Austral. Math. Soc. 6 (1966), 470-476.

[10] H. L. Manocha and B. L. Sharma, "Some formulae by means of fractional derivatives," Compositio Math. 18 (3), (1967), 229-234.

[11] P. C. Munot, “On Jacobi polynomials," Proc. Cambridge Philos. Soc. 65 (1969), 691-695.

[12] M. A. Pathan, "On a general triple hypergeometric series," Proc. Nat. Acad. Sci. India 47 (A) I (1977), 58-60.

[13] M. A. Pathan, "Certain formulas involving $F^{(3)}(x, y, z)$," Acta Cienc. Indica Math. 4 (3) (1978), 316-318.

[14] M. A. Pathan, "On some transformations of triple hypergeometric series $F^{(3)}$, Indian $J$. Pure Appl. Math. 9 (4) (1978), 371-376.

[15] M. I. Qureshi, On the study of multiple hypergeometric functions of higher order, Ph.D. thesis, Aligarh Muslim University, Aligarh, India, 1983.

[16] M. I. Qureshi and M. A. Pathan, "A note on hypergeometric polynomials," J. Austral. Math. Soc. Ser. $B 26$ (1984), 176-182.

[17] E. D. Rainville, "The contiguous function relations for ${ }_{p} F_{q}$ with applications to Bateman's $J_{n}^{(u, v)}$ and Rice's $H_{n}(\zeta, p, v), "$ Bull. Amer. Math. Soc. 51 (1945), 714-723.

[18] E. D. Rainville, Special functıons (Macmillan, New York, 1960).

[19] S. O. Rice, "Some properties of ${ }_{3} F_{2}[n, n+1, \zeta ; 1, p ; v]$," Duke Math. J. 6 (1940), 108-119.

[20] B. L. Sharma, "Some formula for Appell functions," Proc. Cambridge Phllos. Soc. 67 (1970), 613-618.

[21] R. L. Shively, On pseudo Laguerre polynomials, Ph.D. dissertation, University of Michigan, 1953. 
[22] H. M. Srivastava, "Hypergeometric functions of three variables," Ganta 15 (2) (1964), 97-108.

[23] H. M. Srivastava, "Some integrals representing triple hypergeometric functions," Rend. Circ. Mat. Palermo (2) 16 (1-3) (1967), 99-115.

[24] H. M. Srivastava, "Generalized Neumann expansions involving hypergeometric functions," Proc. Cambridge Philos. Soc. 63 (1967), 425-429.

[25] H. M. Srivastava, "Some integrals representing triple hypergeometric functions," Math. Japon. 13 (1) (1968), 55-69.

[26] H. M. Srivastava, "Certain formulas involving Appell functions," Comment. Math. Univ. St. Pauli 21 (1) (1972), 73-99.

[27] H. M. Srivastava, "Certain formulas associated with generalized Rice polynomials-II," Ann. Polon. Math. 27 (1972), 73-83. 